\title{
Le brunch et son réseau d'anglicismes : étude sur un corpus spécifique
}

\author{
Ramón Martí Solano ${ }^{1}$ \\ Universidad de Limoges
}

\begin{abstract}
RÉSUMÉ
Un corpus spécifique est un corpus créé ad hoc en fonction de l'objet de recherche. Ce corpus a été créé à partir de textes d'un sous-genre de la presse française des cinq dernières années en ayant comme mot cible l'anglicisme brunch. Le but principal étant de compter avec un corpus dans lequel chaque texte contient au minimum une occurrence de ce terme. Ensuite, nous avons procédé à l'analyse à la fois quantitative et qualitative du lexique associé à brunch avec un intérêt particulier à d'autres anglicismes qui gravitent autour de lui, comme par exemple street food ou foodtruck. II est aisé de constater que l'emploi d'un anglicisme d'un champ lexical spécifique attire vers lui d'autres anglicismes du même champ. Enfin, nous avons comparé les résultats obtenus dans notre corpus avec ceux de la presse française en général et en particulier avec les données issues des archives électroniques du journal Le Monde depuis 1944. Ainsi faisant, nous avons délimité leur origine, évolution, usage et institutionnalisation en français contemporain.
\end{abstract}

Keywords: brunch, corpus spécifique, genre textuel, anglicisme, xénisme, associations lexicales

\section{ABSTRACT}

A specific corpus is an ad hoc corpus compiled for a specific purpose. The present corpus was created by extracting texts of a sub-genre of the French press over the last five year having as the target word the Anglicism brunch. The main objective is to have a corpus in which every single text has at least one occurrence of the target word. Both quantitative and

$1 \quad$ Corresponding author - Faculté des lettres et des Sciences Humaines 39E rue Camille Guérin, 87036, Limoges, France

Email: ramon.marti-solano@unilim.fr 
qualitative analyses concerning other words closely associated to brunch were carried out and particularly concerning other Anglicisms that gravitate around it as for instance street food or food truck. It should be noted that the use of an Anglicism from a specific semantic field attracts other Anglicisms of the same field. The results of this study were compared with the results of the French press, especially with the data found in the online archives of the newspaper le Monde from 1944 to date. By doing so their origin, evolution, usage and institutionalisation in contemporary French have been delimited.

Keywords: brunch, ad hoc corpus, textual genre, Anglicism, foreignism, lexical associations

\section{Introduction}

S'agissant de cuisine on pourrait penser que la France n'a rien à apprendre des autres langues-cultures, même si pendant des siècles le français a accueilli à bras ouverts grand nombre de mots et d'expressions culinaires originaires des pays voisins comme l'Italie (Hope, 1971) mais aussi d'autres contrés bien plus exotiques souvent par l'intermédiaire surtout de l'espagnol (avocat, cacahuète, chocolat, papaye, tomate, etc.) et dans une moindre mesure du portugais, comme avec ananas ou banane (Walter, 1997).

La France et le français ont toujours été les exportateurs de l'une des grandes fiertés nationales: la gastronomie à la française ${ }^{2}$. Le stéréotype de la cuisine d'origine anglosaxonne, soit-elle britannique ou américaine, comme une cuisine grossière, bourrative et peu variée est bien ancré dans la société française. Mais cette société peut être très paradoxale si on tient compte des informations, enquêtes et statistiques qui nous sont délivrées à des périodes régulières sur les habitudes alimentaires des Français concernant, par exemple, l'ingestion massive d'hamburgers par rapport à d'autres pays européens.

Nous savons que les habitudes alimentaires sont, par les temps qui courent, le produit de modes et de tendances qui s'accentuent de plus en plus dans nos sociétés occidentales avides de changements et de nouveautés. Les influences anglo-américaines ont toujours été importantes concernant les loisirs, et plus particulièrement les sports, la musique, les boissons et la mode et ceci depuis le dixhuitième siècle (Humbley, 2004, p. 108) et, cela va sans dire, concernant aussi la nourriture pour ce qu'elle représente de manifestation et de comportement sociaux.

2 Le repas gastronomique des Français a été inscrit en 2010 sur la liste représentative du patrimoine immatériel de l'humanité de I'UNESCO. 


\section{Le brunch, un mot-valise à la mode}

Il a été signalé que la raison la plus importante, actuellement, qui justifierait un grand nombre de transferts lexicaux d'une langue à une autre est liée aux notions de prestige ou de mode (Hoffmann, 2011, p. 130; Onysko, 2004, p. 62; Bechert/Wildgen, 1991, p. 76), voire de snobisme (Hagège, 1987, p. 97) plutôt qu'à des besoins réels pour combler des lacunes lexicales dans les langues. La plupart de ces transferts lexicaux sont des anglicismes, c'est à dire des emprunts à la langue anglaise faits par le français, en l'occurrence, et par la grande majorité des langues du monde.

An anglicism is a word or idiom that is recognizably English in its form (spelling, pronunciation, morphology, or at least one of the three), but is accepted as an item in the vocabulary of the receptor language. (Görlach, 2003, p. 1)

Ainsi, l'anglicisme brunch ${ }^{3}$ est un amalgame lexical, un mot-valise formé à partir de breakfast et lunch par apocope de la première base-source et aphérèse de la deuxième base-source (Renner, 2006, p. 139). D'après l'Oxford English Dictionary son origine se trouverait dans le jargon universitaire britannique. En voici la première occurrence attestée par le prestigieux dictionnaire qui date de 1896 :

Punch 1 Aug. 58/2 An excellent portmanteau word...indicating a combined breakfast and lunch. At Oxford, however, two years ago, an important distinction was drawn. The combination-meal, when nearer the usual breakfast hour, is 'brunch', and when nearer luncheon, is 'blunch'.

II n'est pas surprenant que dans cette première occurrence il s'agisse d'un emploi métalinguistique de brunch qui va encore plus loin que la simple explication du motvalise mais qui inclut aussi de l'information supplémentaire concernant la distinction brunch / blunch en fonction de la proximité horaire du petit-déjeuner (brunch) ou du déjeuner (blunch). Ce deuxième terme n'a pas eu le succès du premier qui, lui, s'est imposé d'abord en anglais et est devenu aujourd'hui un mot à caractère international au même titre que d'autres mots du domaine culinaire empruntés à l'anglais britannique (pudding), à l'anglais américain (burger), au français (foie-gras) ou à l'italien (pizza).

Le brunch est associé à un repas souvent copieux, composé de mets variés

Il a été avancé que tous les anglicismes en français qui finissent par -unch sont traités comme des noms au masculin, comme par exemple punch, crunch, lunch et brunch (Hugou, 2015, p. 135). 
combinant des aliments et de boissons propres à la fois au petit déjeuner et au déjeuner, ce qui lui confère une entité hybride et cumulative et contribue à son analysabilité lexicale. D'ailleurs brunch est classé haut dans l'échelle de typicalité par rapport à d'autres mots-valises tel que sitcom (Renner, 2006). Même s'il est vrai que la création fait partie à l'origine du parler des jeunes universitaires britanniques à la fin du dix-neuvième siècle, le mot est aujourd'hui plutôt associé à des modes de vie des grandes villes et à des âges qui n'étaient sûrement pas considérés jeunes il y a plus d'un siècle.

En France, l'apparition de brunch dans le journal Le Monde est évidemment plus tardive : son premier emploi correspond au 30 mai 1975 et avait été utilisé, comme c'est souvent le cas pour les premières occurrences, de façon métalinguistique :

Un repas plus consistant qu'un "breakfast", plus léger qu'un "lunch" : une sorte de "brunch" américain de très haute qualité, servi dans une nouvelle vaisselle. Concorde pourra emporter une tonne de matériel et de victuailles... Cinq à six stewards et hôtesses qui ne seront pas...

L'explication sociolinguistique de cet emploi en français n'est autre que l'occasion pour les hôtels, restaurants, bars, cafés et d'autres adresses commerciales de proposer une alternative au déjeuner dominical traditionnel dirigée principalement aux jeunes (et moins jeunes aussi) qui ont fait la fête le samedi soir et la grasse matinée le dimanche matin. Le succès de cette formule réside en la combinaison de mets et de boissons propres au petit-déjeuner américain avec des salades, sandwichs et d'autres plats habituellement consommés dans le repas de midi. C'est donc l'amalgame, blending en anglais, qui fonctionne bien ici, et non exclusivement sur le plan lexicologique. Les young urban professionals, ou yuppies, des jeunes professionnels citadins des années 80 du siècle dernier aux Etats-Unis en étaient la cible privilégiée. Cette population jeune, active, indépendante et libre de contraintes familiales était en quête d'alternatives, de nouveautés et surtout prête à dépenser. Le yuppie voulait se faire plaisir toujours dans une optique qui alliait les produits bons pour la santé avec une vie sociale effervescente et bien remplie. La ville de New York et d'autres grandes agglomérations urbaines y étaient pour beaucoup. Comme d'autres phénomènes de mode, le brunch était à l'époque aux Etats-Unis un produit cent-pour-cent citadin.

Le mot apparaît dans le Dictionary of European Anglicisms même si dans l'entrée il est indiqué que son emploi est assez marginal (Görlach, 2001, p. 38) ${ }^{4}$. Sur les 16 langues

4 Même si la date de la première publication du dictionnaire de Görlach est 2001, il faut savoir que le projet a commencé en 1993 et que l'intention du dictionnaire était de 
européennes représentées dans la grille qui accompagne chaque entrée, seulement trois cases, correspondant au néerlandais, au finnois et au grec, apparaissent en blanc, ce qui veut dire qu'il est totalement intégré (fully accepted), 9 cases sont rayées, dont le français et l'espagnol ${ }^{5}$, signifiant que son intégration est partielle (restricted use) et enfin 5 langues, dont le portugais, avec les cases en noir pour indiquer que cet anglicisme est inconnu (not part of the language). L'état actuel d'intégration, voire d'institutionnalisation, de brunch est tout à fait différent: il fait partie de la nomenclature du Grand Robert (LGR), même s'il n'apparaît pas encore dans le Trésor de la langue française informatisé (TLFi), et est devenu un terme connu et assez répandu parmi les locuteurs francophones en Europe. Quant aux autres langues européennes il semble s'y être installé plus ou moins confortablement non seulement en tant qu'emprunt lexical à l'anglais mais surtout en tant que manifestation incontestable du phénomène de la mondialisation. En revanche, les anglicismes food truck et street food ne sont pas enregistrés dans le dictionnaire de Görlach car il s'agit de phénomènes assez récents dans le domaine des modes culinaires et des habitudes alimentaires et par conséquent pas du tout répandus à l'époque de l'élaboration de ce dictionnaire.

Il convient de signaler que brunch en anglais désigne un repas mais que le mot est lié directement à une mode, à une forme de consommation inséparable de certains types de lieux (hôtels, restaurants, etc.), d'où l'importance d'autres anglicismes, principalement des adjectifs comme cosy, qui vont décrire l'allure et l'ambiance des endroits où le brunch est servi.

Du point de vue morphologique, le pluriel -es rajouté aux noms anglais finissant par -ch n'est pas utilisé régulièrement en français qui préfère, lorsqu'il s'agit du traitement morphologique de ce type d'anglicismes, les formes lunchs et sandwichs à lunches et sandwiches (Humbley, 2004, p. 116). Ceci est aussi le cas pour l'anglicisme brunch dont nous avons trouvé 48 occurrences de la forme brunchs et aucune de la forme brunches dans notre corpus, ce qui corrobore l'observation précédente de Humbley.

documenter l'influence lexicale de l'anglais sur les autres langues européennes jusqu'à 1995 (Görlach, 2001, p. xvi).

5 Le caractère restreint de l'usage de brunch en espagnol pour la même période est corroboré par le fait que ce mot ne fait pas partie de la nomenclature de l'ouvrage Anglicismos hispánicos (Lorenzo, 1996). 


\section{La néologie lexicale, l'emprunt et les xénismes}

Il peut paraître une évidence mais il est aisé de rappeler que le répertoire lexical des langues est en constant renouvellement. Cette affirmation est applicable non seulement en synchronie: le lexique des langues s'est construit et a augmenté considérablement au fil du temps principalement par dérivation lexicale interne mais surtout grâce aux différents mécanismes néologiques dont l'emprunt.

Il convient de signaler que, s'agissant de néologismes liés à des modes et à des tendances, on est face à une néologie spontanée et non à une néologie planifiée ou institutionnelle puisque ces néologismes ont un composant linguistique mais aussi un composant psychosociologique et plus particulièrement, et dans le cas qui nous occupe, un composant socioculturel. II ne faut pas confondre la néologie lexicale avec le changement linguistique : il n'y a pas de changement linguistique dans le cas de brunch et par conséquent pas d'effacement, voire de substitution d'un terme par un autre, mais plutôt un néologisme qui est le résultat d'un emprunt direct à l'anglais dont seulement la prononciation et la morphologie sont adaptées.

Dans cette situation langagière il existe un moment de réception suivi d'un autre beaucoup plus important dans le temps de circulation. Ensuite se produit la stabilisation ou institutionnalisation du néologisme. Quant à brunch on peut parler d'un néologisme référentiel, d'une entité nommée, d'un néologisme nécessaire avec une justification sociale importante, s'agissant donc d'une unité culturelle. Enfin, l'emploi de certains néologismes accorde une touche de prestige social qui est directement liée à l'étrangeté inhérente au mot pour les autres locuteurs et à sa représentation dans l'imaginaire collectif.

II y a emprunt, par conséquent, lorsque l'élément lexical étranger est introduit et maintenu dans le système de la langue par des locuteurs de langue maternelle hors des conditions d'interférence. C'est ainsi qu'on dira que les mots du lexique « français » parking, design, poster, etc. sont des emprunts à l'anglais, parce que ces mots sont utilisés même - et surtout par la masse des francophones qui ne connaissent pas l'anglais et qui ne sont jamais (ou quasiment jamais) en situation de communication bilingue français/anglais. C'est donc au niveau de l'emploi par les locuteurs unilingues qu'il convient de s'interroger sur le statut et les fonctions de l'emprunt. (Pergnier, 1988, p. 114)

Le néologie, comme d'ailleurs l'emprunt, est un phénomène de courte durée: une fois le terme accepté, institutionnalisé et lexicalisé, il devient un mot faisant partie du répertoire lexical des locuteurs d'une langue même s'il garde toujours son caractère d'emprunt de par sa particularité phonologique: concernant le mot 
brunch, le son vocalique particulier à l'anglais $/ \mathrm{N} /$ et la prononciation du digramme $c h$ prononcé à l'anglaise en sont les signes distinctifs et marquent ainsi son caractère d'anglicisme.

Quant aux xénismes ${ }^{6}$, la situation est tout à fait différente. II s'agit de mots étrangers qui ne font pas partie du stock lexical de la majorité des locuteurs unilingues d'une langue donnée et qui, par conséquent, restent inconnus. On ne peut donc parler d'emprunt concernant des mots tels que gravy, take away, tea time, organic, healthy, etc. car il s'agit tout simplement de l'introduction de mots et d'expressions étrangers qui soit nomment des réalités culturelles liées à une langue, comme avec gravy, soit sont le reflet d'une volonté consciente d'utiliser un mot anglais à la place de son équivalent exact en français, comme avec organic («bio ») ou healthy (« sain »). En tout cas, la distinction entre emprunt et xénisme est dépendante du niveau de langue de chaque locuteur : l'écrivain Michel Houelbecq utilise les mots arty et hype ${ }^{7}$ dans ses romans alors que pour la plupart des locuteurs francophones unilingues ces deux mots sont deux grands inconnus.

\section{Corpus et méthodologie}

Nous avons voulu décrire ces objets linguistiques qui sont les néologismes ou les emprunts par le biais de méthodes empiriques en utilisant des techniques d'analyse à la fois quantitatives et qualitatives. Cette approche méthodologique prétend analyser, entre autres, les patrons lexico-syntaxiques qui sont propres à l'ensemble des anglicismes qui apparaissent dans les textes associés au mot pivot brunch. Le choix de cet anglicisme comme mot cible n'est pas anodin : il représente dans la société française actuelle, et plus particulièrement dans les grandes et moyennes villes de ce pays, l'une des tendances les plus importantes d'habitudes alimentaires en lien avec les loisirs.

Notre corpus est composé d'un ensemble d'articles extraits des journaux et

$6 \quad$ En anglais on emploie le terme foreignism et en espagnol extranjerismo.

7 En voici les deux citations tirées des exemples fournis par Le Grand Robert pour chacun des mots: "Je ne le vois pas tellement dans la communication d'une entreprise de voyages ; plutôt dans un truc plus prestigieux, plus arty. Là il va s'ennuyer, il ne va pas rester. » (Plateforme, p. 209). "J'avais appris de Léa que Koh Samui n'était pas seulement un paradis tropical, mais aussi un endroit plutôt hype. À chaque nuit de pleine lune, dans la petite île voisine de Koh Lanta, se déroulait une rave gigantesque ; des gens venaient d'Australie ou d'Allemagne pour y participer. » (Plateforme, p. 94) 
magazines suivants : Paris Match, Le Parisien, Le Parisien Magazine, Le Figaro, Le Figaro Magazine, Madame Figaro et Le Monde Magazine. Les articles choisis du Parisien et du Figaro proviennent de la rubrique «style». Nous avons choisi un échantillon qui nous semble représentatif des sources écrites susceptibles de contenir le mot cible et d'autres anglicismes y associés dans des contextes essentiellement liés aux loisirs, au temps libre, aux habitudes alimentaires, etc. Le concept de genre textuel nous semble fondamental pour la recherche lexicologique en général et pour la recherche sur les emprunts ou les anglicismes en particulier. Certains genres textuels, comme les informations sur la mode, la musique ou les loisirs peuvent foisonner d'anglicismes tandis que d'autres en auront moins ou très peu, comme par exemple les textes juridiques.

Selon une étude de 1977, on ne trouve dans le journal Le Monde, de janvier à mai 1977, qu'un mot anglais sur 166. En outre, le mouvement est très inégalement réparti selon les genres, les registres de langue, les couches sociales, les thèmes d'entretien: la langue de la réclame content beaucoup plus de mots anglais que celle du droit canon ou de l'œnologie : la langue de la critique d'art ou de la littérature en offre beaucoup moins que celles de l'informatique ou de la banque. (Hagège, 1987, p. 74)

Notre corpus fait un total de 305420 mots avec 592 occurrences de brunch, brunchs et bruncher. Nous avons trouvé dix-sept occurrences du verbe bruncher à l'infinitif, deux occurrences de la forme de l'impératif brunchez et une seule occurrence du verbe brunchiser, ce qui fait de ce dernier un hapax dans ce corpus.

Quoi qu'il en soit, il semble que les verbes empruntés directement à l'anglais soient plus nombreux que les adjectifs emprunts directs. De même, on relève des exemples de verbes de type anglo-saxons empruntés et conjugués, contrairement aux adjectifs anglo-saxons, jamais accordés au pluriel ni au féminin. Cette présence de verbes empruntés semble être en contradiction avec le constat des années 50 et 60, lorsque les verbes anglais étaient surtout empruntés sous la forme d'une nominalisation (soit rerecording pour le verbe to rerecord), conjuguée au moyen d'un verbe support (faire un rerecording) (Humbley, 1974). (Humbley, 2010, p. 9)

La création de verbes à partir de noms empruntés à l'anglais est une pratique fréquente en français : le verbe « luncher » est attesté depuis 1928 est répertorié par le TLFi et LGR. En revanche, bruncher est une création relativement récente et non attestée par lesdits dictionnaires. Cette catégorie grammaticale est, de par la nature de la thématique choisie, très peu représentée dans nos résultats: nous avons trouvé le verbe «relooker », dérivation française sur un mot anglais et totalement intégré en français. 
Nous avons commencé notre recherche à partir de la liste de mots produite par le concordancier AntConc, dont nous avons exclu les mots outils, le mot cible brunch et d'autres mots pleins tels que Saint, qui fait référence à des noms de rue de Paris comme la rue Saint-Honoré ou le boulevard Saint-Antoine ou même au canal SaintMartin. Le résultat de ce premier tri donne deux gagnants sans équivoque : "Paris » avec 923 occurrences et « carte » avec 730, ce qui montre que dans notre corpus il s'agit bien d'un phénomène citadin et culinaire. Le mot "dimanche » apparaît en douzième position avec 329 occurrences signalant ainsi le caractère dominical de cette importation américaine. Si à ce score on ajoute les 368 occurrences de l'abréviation "dim.», ce sont 697 occurrences, ce qui fait de «dimanche »le troisième mot plein le plus représenté dans ce corpus.

Les tris à gauche et à droite du mot pivot permettent de repérer de façon claire et rapide des phénomènes lexico-grammaticaux qui pourraient passer autrement inaperçus. Le tri à droite donne divers résultats intéressants : sur un ensemble de 513 occurrences du mot brunch, les combinaisons avec "dimanche ", «samedi » ou « week-end » font un total de 115 occurrences, ce qui représente une fréquence relative de $22,4 \%$. Les combinaisons avec « carte » et « buffet » ne représentent que huit et sept occurrences respectivement comme l'illustre le Tableau 1.

\begin{tabular}{lc}
\hline Structures lexicales & Nombre d'occurrences \\
\hline $\begin{array}{l}\text { brunch le dimanche, brunch du } \\
\text { dimanche, brunch dominical, } \\
\text { brunch le dim., brunch les dim. }\end{array}$ & 68 \\
\hline $\begin{array}{l}\text { brunch le week-end, brunch du } \\
\text { week-end, brunch les sam. et dim., } \\
\text { brunch sam. et dim. }\end{array}$ & 37 \\
\hline $\begin{array}{l}\text { brunch du samedi, brunch le sam., } \\
\text { brunch sam. }\end{array}$ & 10 \\
\hline brunch à la carte & 8 \\
\hline brunch buffet & 7 \\
\hline
\end{tabular}

Tableau 1. Tri à droite de brunch (structures lexicales les plus fréquentes)

En revanche, le tri à gauche ne proportionne pas de données réellement intéressantes: les colligations contiguës les plus représentatives quantitativement se font avec les déterminants défini et indéfini, «le » et « un » respectivement, et donc n'apportent pas vraiment d'informations sur leur environnement lexical.

Lorsque brunch ne rentre pas dans les patrons présentés dans le Tableau 1 il se décline de différentes façons comme l'illustrent les exemples suivants : 
(1)

(2)

(3)

(4)

(5)

(6)

Le brunch. II se veut organic et est surtout pensé par un chef qui s'active, Christophe Hay.

Récemment reprise par Sylvestre Wahid - fraîchement gratifié de deux étoiles au Michelin pour sa table Sylvestre à l'étage - la brasserie de I'hôtel Thoumieux propose depuis quelques semaines un brunch top qualité.

En août, forfait du samedi au jeudi : 4180 comprenant 5 nuits en grande chambre double de luxe avec petits déjeuners, le brunch et le dîner gastronomique du dimanche.

Déjà presque classique, cette tarterie pas tarte du tout, aux allures rustique chic pour du tout fait maison. À vérifier avec le brunch servi à table, joli défilé de tarte salée, puis sucrée, de salade de fruits, scones et müesli, avec ce qu'il faut de jus de fruits frais et de boissons chaudes.

Pour vous distraire, pléthore d'activités branchées, de corners mode en beauty bar, d'expos de street art en vide-dressings, d'ateliers et jeux pour les enfants en clubbing. Côté food, le brunch de la chef de l'excellent CheZAline, des burgers bio, et des food corners en veux-tu en voilà. Autre possibilité, le Brunch Market à Confluences, lieu plurisdisciplinaire [sic] du XXe, avec confitures Coline Boileau, bijoux, vêtements et accessoires mode et maison.

Quoi? Jordan entend combiner la gastronomie US avec les produits français. Tout est à base d'ingrédients bio, en provenance de producteurs de Rhône Alpes et du Pays de la Loire et Poitou. Préférez I'un des quatre burgers aux «carnitas tacos» (épaule de porc dans des tortillas maison), un peu moins réussis. Pour le brunch et le dessert, cupcakes, carrot cakes et pancakes vous tendent les bras.

Une première analyse de cette sélection d'exemples fait ressortir le lien étroit entre brunch et la nourriture bio et saine - exemples (1), (6) et (7) - mais aussi entre brunch et le luxe et le chic - exemples (2), (3) et (4) - comme le montrent les mots et groupements de mots soulignés: "organic », "deux étoiles au Michelin », «top qualité », «luxe », « gastronomique », «chic », «bio ». De son côté, l'exemple (5) est particulièrement représentatif de ce type de textes qui foisonnent d'anglicismes (dans son environnement lexical on trouve « branchées », " corners », " beauty bar », «street art », « vide-dressings », « clubbin », « food » et « food corners »).

Des recherches sur le lemme et son champ dérivationnel, en l'occurrence brunch, brunchs et bruncher font ressortir d'autres anglicismes qui gravitent autour de ceuxci et qui, par ce fait, sont facilement repérables puisqu'ils se trouvent dans l'environnement lexical proche. Ils peuvent être très variés et vont des desserts, 
gâteaux et d'autres préparations sucrés ou salés tels que jelly, scone, muffin ${ }^{8}$ ou chip à d'autres termes tels que after dans after-brunch, after work, after parties et des créations hybrides telle qu'en after.

\begin{tabular}{ll}
\hline Lexèmes & Nombre d'occurrences \\
\hline pancake & 33 \\
\hline cheesecake & 24 \\
\hline scone & 21 \\
\hline granola & 16 \\
\hline cookie & 16 \\
\hline muffin & 15 \\
\hline bagel & 14 \\
\hline fish and chips & 14 \\
\hline carrot cake & 13 \\
\hline cupcake & 8 \\
\hline club sandwich & 7 \\
\hline brownie & 6 \\
\hline
\end{tabular}

Table 2. Tri à droite de brunch (champ lexical d'anglicismes alimentaires par ordre de fréquence)

A l'exception de scone et fish and chips, associés à la Grande Bretagne, le Tableau 2 montre la prédominance des mets typiquement américains: le pancake, en première position, évoque le petit déjeuner américain, ce qui est connu comme pancake breakfast, un petit déjeuner à base de crêpes épaisses accompagnées de saucisses et de bacon. Lorsque le mot thé est utilisé en anglais, tea, il s'associe de préférence avec british [sic] et time comme l'illustrent les exemples suivants: "Un mélange de high tea british », «à savourer comme un five o'clock tea! », " entre une expo et un tea time dans un palace ». Les anglicismes pancake, cheese-cake, scone, cookie, muffin, bagel, cupcake et brownie sont attestés dans Le Grand Robert : ils sont des préparations ou des produits bien connus et ne posent pas de problème de

8 Le mot muffin a deux référents très distincts en anglais. D'une part il existe ce qu'aux EtatsUnis on dénomme English muffin, qui est, d'après le Trésor de la langue française informatisé (TLFi], un «[P]etit pain moulé, rond, à croûte mince et pâle, consommé surtout en Angleterre et que l'on sert (grillé et beurré) avec le thé. » D'autre part, il existe un autre référent, celui-ci d'origine américaine, qui désigne un grand biscuit rond individuel souvent agrémenté de myrtilles (blueberry muffin) et associé aux modes alimentaires des Etats-Unis et du Canada. Il est intéressant de noter que ce deuxième sens n'apparaît pas dans le TLFi alors que c'est celui-ci le plus usité et le premier qui vient à l'esprit aux locuteurs francophones. Le Grand Robert n'enregistre que le sens de «Petit pain rond cuit dans un moule, qui se mange en général grillé et beurré ». 
décodage à la plupart des locuteurs francophones unilingues.

\section{Anglicismes adjectivaux}

Une requête en utilisant le morphème dérivationnel adjectival -y et qui s'appuie de manière inductive sur la morphologie lexicale des deux langues nous a permis de repérer un nombre important d'anglicismes qui ont un lien plus ou moins direct avec brunch. Ils sont en nombre de dix-huit : arty, bakery ${ }^{9}$, blue navy, cosy, curry, easy, flashy, gay-friendness ${ }^{10}$, girly, gravy, grey, healthy, jazzy, jelly, party, punchy, take away, trendy. La plupart sont des hapax ou des anglicismes avec un très faible degré de représentativité. En revanche, voici les cinq anglicismes - quatre adjectifs et un nom - le plus représentés avec le nombre d'occurrences entre parenthèses :

- $\operatorname{arty}(27)$

- $\operatorname{cosy}(24)$

- healthy (17)

- take-away (12)

- trendy (11)

Il est intéressant de noter l'engouement pour le mot healthy dans ce contexte, anglicisme qui n'est pas courant dans la langue parlée quotidienne contrairement à ce qui se passe avec cosy ou flashy qui, eux, sont d'un usage très répandu et normal parmi les locuteurs en France - cosy est souvent utilisé pour désigner un intérieur où on se sent bien grâce à sa décoration douce et enveloppante alors que flashy s'emploie pour désigner des couleurs très vives ou éclatantes.

Comment expliquer l'explosion du mot arty qui, lui, n'est d'habitude pas utilisé dans la langue orale et qui gagne du terrain dans ce contexte ? L'adjectif arty est un dérivé

9 On pourrait aussi se poser la question sur le grand nombre d'occurrences d'un anglicisme tel que bakery (23) dans le pays de la baguette qui se vante, à juste titre, du nombre de boulangeries mais surtout de la qualité du pain. En réalité, lorsqu'on observe les occurrences on se rend compte que celles-ci correspondent presque toutes à la même entité nommée, concrètement à une boulangerie du neuvième arrondissement de Paris qui porte son nom en anglais. II faut par conséquent ne pas en tenir compte dans l'analyse.

10 Nous avons inclus ce terme dans notre sélection même s'il s'agit d'un composé dont la terminaison n'est pas en $-y$ : c'est le premier élément du composé qui finit par $-y$. II s'agit d'un pseudo-anglicisme morphologique (Furiassi, 2010, p. 31) puisque le terme utilisé en anglais est gay-friendliness. 
relativement récent dans la langue anglaise : la première occurrence écrite attestée par l'OED date de 1901. L'adjectif fut utilisé d'abord pour décrire du mobilier ou de la décoration pour ensuite être appliqué à des personnes avec des prétentions plus ou moins artistiques. II a une fréquence très basse autant dans le BNC que dans le COCA. Dans notre corpus il est utilisé principalement soit en collocation avec « faune », «clientèle » ou « jeunesse » soit avec « décor», «loft» ou « spots ». Dans ce sens-là rien ne différencie ces collocations françaises des bases avec lesquelles le collocatif arty se combine en anglais même si dans cette langue la préférence est donnée à des bases telles que person, chap, type, crowd et party, c'est-à-dire à des individus ou à des groupes d'individus plutôt qu'à des lieux. II est assez symptomatique que le brunch français soit associé à ce concept qui dénote en anglais quelqu'un qui est, ou plutôt voudrait paraître, très intéressé par tout ce qui est en rapport avec l'art et le milieu des artistes. Le mot arty prend souvent une connotation négative en anglais et peut être utilisé péjorativement tandis qu'en français son emploi ne l'est pas. On pourrait parler d'une équivalence trompeuse dans le sens où le mot est connoté négativement en anglais et positivement en français. Cette même observation peut se faire concernant d'autres anglicismes, par exemple trendy lorsqu'il est appliqué à des personnes. Le transfert lexical de ces deux adjectifs en français s'accompagne d'un processus de changement sémantique concernant leur valeur axiologique.

De son côté, l'anglicisme healthy entre en collocation soit avec des noms de lieu tels que "cantine » ou " points de chute » soit avec des noms de nourriture tels que "gâteaux» ou «salades» comme l'illustrent les exemples suivants: "C'est la cantine healthy du quartier », "I'un de nos points de chute healthy préférés », "des gâteaux healthy sans gluten» et "pour une salade healthy». Les collocations privilégiées en anglais sont par ordre de fréquence dans le COCA people, eating, food, lifestyle, diet, weight et children mais en aucun cas des noms de lieux et très rarement des noms des plats : healthy salad n'a que six occurrences dans le COCA, ce qui est extrêmement peu pour que cette combinaison soit prise en compte comme une combinaison courante en anglais.

Si l'on compare le comportement collocationnel des mots trendy et «tendance » dans notre corpus, on s'aperçoit que c'est l'anglicisme qui s'associe préférentiellement à d'autres mots dans une relation lexicale de collocation. De son côté le mot «tendance » suit plutôt le comportement propre à l'usage classique de ce substantif mais avec quelques exceptions telles que «dans ce restaurant tendance », "devenir un véritable Parisien tendance » ou même "ce fabricant de jouets épurés et ultratendance ». En revanche, les combinaisons lexicales de trendy présentent une configuration tout à fait différente: "autre night-club trendy 
parisien », " C'est un concept trendy », « la (longue) liste des lieux trendy », " ce lieu trendy », « une clientèle jeune et trendy », «la faune trendy». Comme le montrent les exemples extraits de notre corpus, trendy se combine principalement avec des mots qui désignent des lieux et des gens, exactement comme le mot se comporte en anglais où les collocatifs privilégiés sont : restaurants, clothes, boutiques et shops ${ }^{11}$. La collocation night-club trendy du premier exemple est hybride du fait qu'elle suit l'ordre syntaxique normal du français contrairement à de la collocation anglaise trendy night-club.

D'un point de vue morphosyntaxique les résultats obtenus confirment l'emploi d'anglicismes qui sont des adjectifs insérés dans une configuration linguistique typiquement française. Ainsi, organic dans « sa gamme exceptionnelle de produits organic ", british ${ }^{12}$ dans "Le temple de l'organic food british », voire une combinaison d'adjectifs tels organic et healthy dans « Rendez-vous organic et healthy prisé des foodies parisiens ».

\section{Autour de food}

Le mot pivot food a été utilisé pour repérer toutes les différentes combinaisons concernant des mots composés, mots dérivés, anglicismes hybrides et d'autres créations néologiques. Nous en avons trouvé 145 occurrences dont les cas suivants : food truck, street food, foodista, foodies, foodeuse, foodocratie, foodentsia, junk food, seafood, fast-food, food addict, food corner, food truck gourmet, finger food. Quelques-uns de ces mots sont des hapax, comme par exemple foodentsia et foodocratie mais ils sont intéressants du point de vue de la créativité lexicale propre au style journalistique. Ce sont food truck et street food, avec 35 et 22 occurrences respectivement, qui occupent les premières places dans cette catégorie, ce qui fait de ces composés de nouveaux concepts liés aux habitudes alimentaires branchées ou à la mode. Les exemples ci-dessous montrent l'emploi en contexte de ces deux composés:

Il sera bientôt inutile de prendre l'Eurostar en cas d'envie incontrôlable de fish and chips, le casse-croûte traditionnel du Londonien. A compter de juin, un food truck aux couleurs de l'Union Jack sillonnera la

11 Nous avons utilisé les deux grands corpus généraux de la langue anglaise, à savoir le British National Corpus (BNC) et le Corpus of Contemporary American English (COCA) pour rendre compte des collocatifs privilégiés en anglais.

12 Toutes les occurrences de british dans notre corpus (16 en total) sont en minuscule. 
banlieue proche de Paris pour délivrer ses cornets imprimés papier journal.

(8) La tendance du food truck de qualité, venue des Etats-Unis, et très présente à Londres, débarque à Paris en novembre 2011, quand Kristin Frederick déboule avec son camion à burgers géniaux, que l'on suit à la trace via les réseaux sociaux.

(9) De la vraie « street food » qui enchantera les bobos dans sa dimension cosmique, et les grandes personnes.

(10) Jusqu'à la fin du mois de juin, les Yvelines accueillent le gratin de la street food parisienne, avec le premier Food Trucks Festival.

La street food continue de grignoter le canal Saint Martin avec cette cantine branchée ouverte depuis août par deux jeunes femmes d'origine arménienne.

Les exemples ci-dessus montrent bien le lien entre ces deux concepts récents et le Royaume-Uni (7) et les Etats-Unis (8). II existe aussi une relation évidente avec la capitale et les milieux branchés et bobos - exemples (9), (10) et (11).

Nous avons trouvé sept occurrences de foodies, toutes au pluriel, parmi lesquelles l'exemple suivant:

$$
\begin{aligned}
& \text { Au bar à vins Frenchie de Gregory Marchand (5-6, rue du Nil, Ile, tél.: } 01 \\
& 403996 \text { 19), on peste contre la hype ambiante et les foodies british } \\
& \text { surexcités qui font la queue devant l'entrée à } 19 \text { heures pétantes. }
\end{aligned}
$$

Ce terme est utilisé en anglais pour désigner une personne avec un intérêt particulier pour la nourriture, particulièrement par les différentes variétés de cuisine et de nourriture, à la différence de "gourmet» qui désigne un connaisseur et amateur de haute cuisine. Notre corpus a donné aussi huit occurrences de foodistas, toutes au pluriel, parmi lesquelles ces deux exemples :

Relifté fin 2013, le «mall» des foodistas et des gourmets ne manque pas d'atouts avec ses $4500 \mathrm{~m}^{2}$ d'espaces divers entièrement dédiés à la gastronomie dont un marché richement garni.

On ne vous refait pas le topo sur Bertrand Grébaut et son Septime surbooké de people et foodistas.

L'anglais américain emprunt le mot barista de l'italien dont la première occurrence dans l'OED est de 1982. D'après le même dictionnaire le mot fashionista serait un emprunt du suffixe espagnol -ista sur un étymon anglais fashion dont la première occurrence est de 1992. Tous deux sont également associés à l'anglais américain. Foodista s'agit par conséquent d'une création néologique du français par analogie avec probablement fashionista dans le sens de a devotee of the fashion industry ou 
même a wearer of high-fashion clothing.

\section{Fréquence et vitalité dans la presse française généraliste}

Il est connu qu'un grand nombre d'anglicismes sont spécialement utilisés dans les médias en général et dans la presse écrite ou électronique en particulier. La presse se révèle l'endroit privilégié non seulement pour l'utilisation d'anglicismes mais aussi pour sa diffusion (Pulcini et al., 2012, p. 19-20).

Nous avons voulu comparer la présence des anglicismes les plus représentés dans notre corpus avec leur présence dans la presse française généraliste et plus particulièrement dans le journal Le Monde. "Bien que le nombre d'études sur les anglicismes en français soit plus modeste, rien n'empêche le linguiste d'aujourd'hui de prendre comme corpus Le Monde, quotidien de référence pour la France, et disponible depuis longtemps sous forme de CDROM annuel. » (Humbley, 2010, p. 10).

La possibilité d'avoir accès aux archives électroniques depuis 1944 permet de se faire une idée plus précise de l'implantation d'un certain nombre d'anglicismes.

\begin{tabular}{lll}
\hline Anglicismes & Nombre d'occurrences & Année de la première occurrence \\
\hline arty & $345^{13}$ & 1986 \\
\hline brunch & 277 & 1975 \\
\hline fooding & $117^{14}$ & 2000 \\
\hline trendy & 80 & 1981 \\
\hline street food & 65 & 2006 \\
\hline food truck & 54 & 2011 \\
\hline
\end{tabular}

Tableau 3. Echantillon d'anglicismes dans Le Monde.

D'après les résultats obtenus dans les archives électroniques du journal Le Monde on constate que le mot brunch est bien représenté depuis 1975. En revanche, les tendances représentées par les concepts de street food et de food truck sont beaucoup plus récentes, 2006 et 2011 pour les premières occurrences respectivement. En ce qui concerne les adjectifs associés, et bien que nous ayons

13 Le nombre d'occurrences réelles sont moindres car le moteur de recherche des archives électroniques du Monde ne tient pas compte de l'orthographe et donc prend en compte le nom propre Arty.

14 Ce chiffre prend en compte le nom d'un site commercial et par conséquent le nombre d'occurrences réels du mot fooding est aussi moins important. 
trouvé quelques occurrences d'Arty en majuscule correspondant à un nom de famille mais également à une marque commerciale, cet adjectif est beaucoup mieux représenté que l'adjectif trendy.

\section{Conclusions}

La création d'un corpus spécifique à partir de sources appartenant aux mêmes genre textuel et période chronologique est un moyen efficace d'examiner la présence et vitalité des anglicismes d'un champ sémantique et des champs sémantiques complémentaires. L'utilisation d'un concordancier permet l'analyse contextuelle à partir d'un mot pivot, brunch en l'occurrence, pour le repérage d'autres anglicismes présents dans son environnement lexical qui sont, à leur tour, utilisés en tant que mots pivots pour des repérages successifs.

II est essentiel de faire la différence entre les concepts d'emprunt et xénisme car nombre de ces unités lexicales ne font pas partie du stock lexical des locuteurs mais sont le produit d'un choix stylistique de la part des journalistes.

Le nombre total d'anglicismes est très important compte tenu de la petite taille du corpus, ce qui s'explique par les critères de compilation et de sélection des sources. II s'avère évident que dans la presse dominicale et plus particulièrement dans celle adressée à un public féminin les anglicismes sont légion lorsqu'il s'agit de la nourriture en tant que loisir et tendance : quelques énoncés regorgent d'anglicismes jusqu'au point de devenir même cryptiques pour des lecteurs non familiarisés avec l'anglais.

Le terme brunch, qui d'après Görlach n'avait vraiment pas sa place à la fois en France et en français dans les années 1980 du siècle dernier, prend un essor aujourd'hui non seulement dans la presse spécialisée mais aussi dans la presse généraliste.

Les nouvelles tendances concernant la nourriture (brunch, food truck, street food, etc.) sont un phénomène plutôt citadin, et plus particulièrement parisien. Nous voulons ici faire l'hypothèse qu'une sélection de la presse régionale aurait donné des résultats assez différents en matière du nombre mais aussi de la diversité d'anglicismes. Même si l'influence culinaire britannique est présente (tea time, fish and chips, scones, etc.), ce sont sans aucun doute les emprunts et xénismes lexicaux américains qui prennent le devant, corroborant ainsi une influence du style de vie des États-Unis en ce qui concernent les tendances par rapport à la nourriture. 


\section{Article history}

Paper received: 25 February 2017

Paper received in revised form and accepted for publication: 19 April 2017

\section{About the Author}

Dr. Ramón Martí Solano is a lecturer in English linguistics at the University of Limoges, France. He read Spanish and English at the University of Seville, Spain, and did an MA in Anglo-Germanic Philology in the same institution. He worked as a Spanish exchange teacher at the University of Harvard, USA. His main field of research is phraseology and his doctoral thesis deals with variation and innovation of phraseological units in the British press. Other research interests include language contact and Anglicisms, contrastive linguistics, paremiology, corpus linguistics, lexical morphology and semantics, lexicography and corpus linguistics. He has collaborated as a proof-reader in the project "Widespread Idioms in Europe and Beyond: A Cross-linguistic and Cross-cultural Research Project". He is a member of the European Society of Phraseology (Europhras) and of the International Association of Paremiology (AIP-IAP). He has published several articles on Anglicisms, paremiology, phraseology, word-formation and lexicography in Spanish, French, and English. He is a member of the research project Aliento (www.aliento.eu) on the circulation of proverbs between the East and the West.

\section{Références}

Anthony, L. (2014). AntConc (Version 3.4.3). Tokyo: Waseda University, <http://www.laurenceanthony.net/> [11/01/2017].

Bechert, J. \& Wildgen, W. (1991). Einführung in die Sprachkontaktforschung. Darmstadt: Wissenschaftliche Buchgesellschaft.

British National Corpus. <http://corpus.byu.edu/bnc/> [15/12/2016].

Corpus of Contemporary American English. <http://corpus.byu.edu/coca/> [15/12/2016].

Furiassi, C. (2010). False Anglicisms in Italian. Milan: Polimetrica.

Görlach, M. (2001). A Dictionary of European Anglicisms. Oxford: Oxford University Press. 
Görlach, M. (2003). English Words Abroad. Amsterdam/Philadelphia: John Benjamins.

Hagège, C. (1987). Le français et les siècles. Paris: Odile Jacob.

Hoffmaan, Z. 2011. Ways of the World's Words. Language Contact in the Age of Globalization. Bern: Peter Lang.

Hope, T. E. (1971). Lexical Borrowing in the Romance Languages. A Critical Study of Italianisms in French and Gallicisms in Italian. Volume I. Oxford: Blackwell.

Houelbecq, M. (2001). Plateforme. Paris: Flammarion.

Hugou, V. (2015). Morphosyntactic adaptation of English loanwords in present-day French: A study of gender and number. Съпоставително езикознание 3, 123-162.

Humbley, J. (1974). Vers une théorie de l'emprunt linguistique. Cahiers de lexicologie 25, 46-70.

Humbley, J. (2004). French. In M. Görlach (Ed.), English in Europe (pp. 108-127). New York: Oxford University Press.

Humbley, J. (2010). Peut-on encore parler d'anglicisme? Lexique, normalisation, transgression (pp.21-45). <https://hal-univ-diderot.archives-ouvertes.fr/hal00967014/ document> [04/01/2017].

Le Grand Robert de la langue française, <http://www.lerobert.com/le-grand-robert/> [15/12/2016].

Le Monde, <http://abonnes.lemonde.fr/> [15/12/2016].

Lorenzo, E. (1996). Anglicismos hispánicos. Madrid: Gredos.

Onysko, A. (2004). Anglicisms in German: from iniquitous to ubiquitous? English Today 20/1, 59-64.

Oxford English Dictionary. <http://www.oed.com/> [04/01/2017].

Pergnier, M. (1988). A propos des emprunts du français à I'anglais. In M. Pergnier (Ed.) Le français en contact avec l'anglais. En hommage à Jean Darbelnet (pp. 113-117). Paris: Didier érudition. .

Pulcini, V., Furiassi, C. \& Rodríguez González, F. (2012). The lexical influence of English on European languages. In C. Furiassi, V. Pulcini \& F. Rodríguez González (Eds.), The Anglicization of European Lexis (pp. 1-24). Amsterdam: John Benjamins.

Renner, V. (2006). Dépasser les désaccords : pour une approche prototypiste du concept d'amalgame lexical. Le Désaccord (pp.137-147) <https://halshs.archivesouvertes.fr/halshs-00512302/document> [04/01/2017].

Trésor de la langue française informatisé. <http://www.cnrtl.fr/definition/> [24/12/2016].

Walter, H. (1997). L'Aventure des mots français venus d'ailleurs. Paris: Laffont. 